\title{
Array of hope for biosensors in Europe
}

\author{
Anthony P.F. Turner
}

While some US commentators have been prematurely mourning the demise of the biosensor field, three important European meetings bear witness that it is alive and kicking. Beginning with the Fifth World Congress on Biosensors*, continuing with a workshop in Luckenwalde, Germany', and finishing with a NATO Advanced Research Workshop $p^{\ddagger}$, speaker after speaker at each meeting emphasized the rich diversity of diagnostic applications to which biosensors can be put to good use'. The World Congress on Biosensors was the largest ever event in the field, with over 400 oral and poster presentations from 47 countries and strong commercial representation in the audience. The message that these meetings brought home? Sensitivity, stability, and diversity will all be pivotal for future biosensor technologies.

Professor Anthony P.F. Turner is head of the Institute of BioScience and Technology at Cranfield University, Cranfield, Bedfordshire, MK43 OAL, UK (A.P.Turner@Cranfield.ac.uk; http://www.cranfield.ac.uk/ibst).

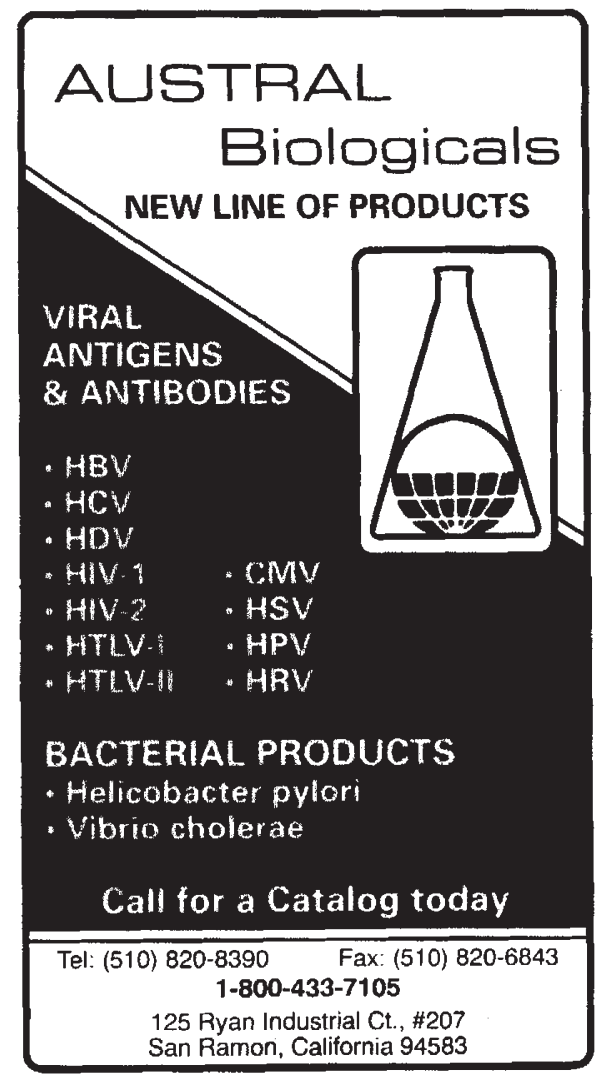

READER INQUIRY NO. 1105
The specificity offered by biological or biologically derived sensing elements in combination with physicochemical transducers, such as optical, electrochemical, thermometric, piezoelectric or magnetic devices, has been long appreciated. This performance has, however, been achieved at a price (i.e., the inherent instability of large proteins). Although enzymes like glucose oxidase have been amenable to commercialization in diagnostic products, such as the mediated amperometric home blood glucose sensors sold by Abbott (Abbott Park, IL), Boehringer Mannheim (Mannheim, Germany), Bayer (Leverkusen, Germany), Lifescan (Milipitas, CA) and Kyoto Daiichi (Kyoto, Japan), other proteins have been more difficult to integrate with microelectronics.

As biosensors move toward the ultimate in sensitivity, the performance of single molecules as receptors must be guaranteed. Likewise, arrays of sensors capable of servicing the demand for wide and flexible menus of analytes, together with the ability to capitalize on advances in chemometrics, will force examination both of the molecular size and of the stability of proposed ligands. All this must be achieved with sufficient diversity to satisfy the appetite for devices that can cope with the data unravelling from the genome sequencing projects (where the impact of phenotypic expression in disease as opposed to genotype has hardly been grasped), the need to understand the complexity of the ecosystem (where probably only $20 \%$ of pollutants are measured, and $50 \%$ can currently be measured), and the less savoury by-products of science such as the threat of terrorist attack using biological weapons, which was graphically described by Fred Milanovich (Lawrence Livermore National Laboratory, CA) at Biosensors ' 98.

One impressive solution to the above problems was furnished by David Baselt's team (Naval Research Laboratory, USA), who won the Biosensors \& Bioelectronics Award for the most original contribution to Biosensors '98. Baselt described their work on the force amplified biological sensor (FABS) and the bead array counter (BARC), which uses antibody-antigen binding interactions to immobilize magnetic microparticles on a solid surface. A variable magnetic field can then be applied to selectively pull away beads bound by nonspecific interactions and/or specific interactions. Force, magnetic field, or optical transducers are used to determine the number of particles that remain on the surface. This elegant approach combines the prospect of ultra-high sensitivity with a practical measurement mode.
Bruce Cornell (Cooperative Research Centre for Molecular Engineering, Australia) picked up the theme of sensitivity combined with stability in a plenary lecture describing his group's research on switching of synthetic ion channels assembled within a lipid bilayer membrane tethered to a gold substrate. He reported sensing units based on the bacterial ion channel gramicidin that could be switched by a range of receptors, including those for antibodies and nucleotides, evolving a vision of single-molecule detection assembled into diverse analytical arrays.

Apart from sensitivity, one of the most striking themes in this summer's congress season, however, was the move toward biomimetic receptors for inclusion in biosensors. This trend began at Biosensors ' 98 , but was amplified particularly strongly at the two workshops that followed. For example, Beining Chen (Cranfield University, UK) described her group's work using combinatorial and computational chemistry to generate novel hexapeptide ligands for glycosylated hemoglobin. The methodology is able to produce synthetic receptors that rival antibody technology, but are also stable, inexpensive, and fully characterized.

Two pioneers of molecular imprinting, Gunther Wulff (University of Dusseldorf, Germany) and Klaus Mosbach (University of Lund, Sweden) both reviewed the field at the Luckenwalde Workshop and the area was picked up again by Kenneth Shea (University of California) and Sergey Piletski (National Academy of Sciences of Ukraine) in Kiev. Overall, there now appears to be a clear tide of belief that biomimetic approaches have come of age.

The powerful combination of methods to generate semisynthetic or synthetic chemical diversity coupled with an advanced understanding of biomolecular interactions is yielding a generation of artificial receptors that take the best performance of biology and build onto it the simplicity and stability associated with traditional products of organic synthesis. We now have the tools to fuel a microanalytical revolution to complement the ubiquitous microprocessor and telecommunications presence. Rumors of our demise have been greatly exaggerated.

*Fifth World Congress on Biosensors, Biosensors '98, Berlin, Germany, June 3-5, 1998; 'Biosensor Workshop, Luckenwalde, Germany, June 5-8; 'NATO Advanced Research Workshop in Kiev, Ukraine, July 6-9.

\footnotetext{
1. A selection of 25 papers from the World Congress on Biosensors will be published in the joumal Biosensors \& Bioelectronics $13(7-8)$ : In press, which will also feature reports of both Biosensors ' 98 and the NATO ARW.
} 